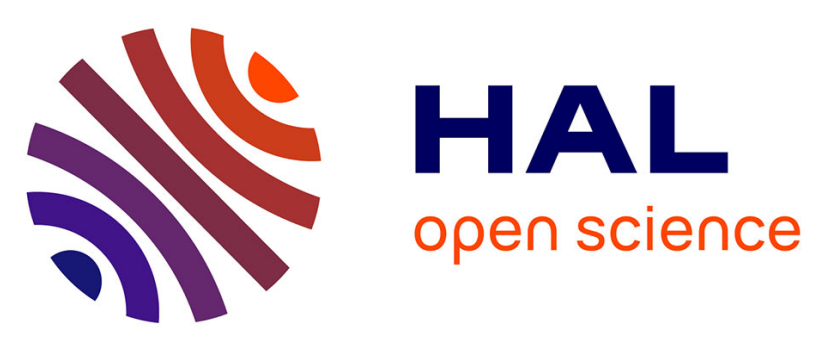

\title{
Rythme d'apparition des racines primaires du maïs (Zea mays L.) I. - Etude détaillée pour une variété en un lieu donné
}

Didier Picard, Marie-Odile Jordan, Roland Trendel

\section{- To cite this version:}

Didier Picard, Marie-Odile Jordan, Roland Trendel. Rythme d'apparition des racines primaires du maïs (Zea mays L.) I. - Etude détaillée pour une variété en un lieu donné. Agronomie, 1985, 5 (8), pp.667-676. hal-00884798

\section{HAL Id: hal-00884798 \\ https://hal.science/hal-00884798}

Submitted on 1 Jan 1985

HAL is a multi-disciplinary open access archive for the deposit and dissemination of scientific research documents, whether they are published or not. The documents may come from teaching and research institutions in France or abroad, or from public or private research centers.
L'archive ouverte pluridisciplinaire HAL, est destinée au dépôt et à la diffusion de documents scientifiques de niveau recherche, publiés ou non, émanant des établissements d'enseignement et de recherche français ou étrangers, des laboratoires publics ou privés. 


\section{Rythme d'apparition des racines primaires du maiis (Zea mays L.) I. - Etude détaillée pour une variété en un lieu donné}

Didier PICARD, Marie-Odile JORDAN \& Roland TRENDEL

I.N.R.A., Station d'Agronomie, F 68021 Colmar

Une étude du nombre de racines séminales et nodales primaires du maïs et de leur rythme d'émission a été effectuée pour une variété, " Dea ", cultivée, selon les normes de la grande culture, sur sol de loess pendant deux années consécutives.

Il est aisé de distinguer l'entre-nœud d'origine des racines nodales primaires même au stade récolte, en raison de l'étagement de ces entre-nœuds et de la variation linéaire du diamètre des racines avec leur rang.

Le nombre de racines nodales primaires commence par diminuer, de 3,7 à l'entre-nœud 2 à 2,2 à l'entre-nœud 4, puis augmente pour atteindre environ 12,4 à l'entre-nœud 8. Aux entre-nœuds 9 et 10, la plupart des racines restent à l'état d'ébauche. Le coefficient de variation du nombre de racines nodales primaires par entre-nœud est fonction des entre-nœuds et des années.

L'émission des racines nodales primaires se fait entre-nœud par entre-nœud. Elle est en relation étroite avec celle des feuilles et dure en moyenne 2 phyllochrones pour chaque entre-nœud.

Mots clés additionnels : Phyllochrone, somme de températures, entre-nœuds.

Rate of appearance of primary roots of maize. I. - Detailed study of one cultivar at one site.

The number, and rate of appearance, of seed and primary nodal roots of maize was studied in cultivar Dea in the field, on loess soil, over 2 successive years. It was easy to identify the internode from which the primary nodal roots originated, even at harvest, because of their spacing and of their linear variation in diameter according to rank. The number of primary nodal roots fell from 3.7 at internode 2 to 2.2 at internode 4 , then rose to 12.4 at internode 8 . At internodes 9 and 10 , most roots remained very short. The coefficient of variation of the number of primary nodal roots per internode depended on internode and on year. The primary nodal roots appeared one internode at a time, their appearance being closely linked with that of the leaves and lasting about 2 phyllochrones for each internode.

Additional key words : Internodes, phyllochrone, sum of temperatures.

\section{INTRODUCTION}

-Etant donné la définition de l'Agronomie et ses relations avec l'Agriculture (cf. notamment SEBILLOTTE, 1974), la connaissance d'un phénomène qui intéresse l'agronome passe le plus souvent par 3 étapes successives : sa mise en évidence, ainsi que celle des facteurs et conditions qui entrent en jeu (approche essentiellement qualitative); la réalisation d'un modèle quantitatif susceptible de l'expliquer a poste- riori ; enfin, la réalisation d'un modèle quantitatif probabiliste, susceptible de le prévoir, ainsi que ses effets. Pour la $2^{e}$ étape et, plus encore, pour la dernière qui n'est pas fréquemment atteinte, la modélisation constitue un outil de travail privilégié.

Les recherches en modélisation de la croissance et du développement du maïs montrent que la structure et la dynamique de l'enracinement de cette plante sont particulièrement mal connues (GARCIA DE CORTAZAR, 1982 ; GIRARDIN, 1982 ; KUNDU et al., 1982). Ainsi, 
les observations de terrain même les plus simples contredisent le modèle de description utilisé. Ceci traduit l'absence de données précises sur l'apparition des racines, leur vitesse d'allongement, leur ramification. En effet, si, par exemple, l'analyse du rythme d'apparition des racines primaires en relation avec le développement des parties aériennes a été tentée au champ, pour le blé (PINTHUS, 1969 ; KLEPPER et al., 1984) ou le riz (PICARD \& JACQUOT, 1976), elle ne semble pas avoir été faite pour le maïs.

Les seuls résultats disponibles (cf. la revue par BONHOMME, 1983) concernent l'évolution de la masse ou de la longueur totale des racines obtenues par des méthodes de carottage (MENGEL \& BARBER, 1974) ou de "planches de fakir" (Allmaras \& NElson, 1971). Or ces données résultent des interrelations entre la croissance de la plante, et particulièrement de ses racines, et les facteurs et conditions de milieu (régime hydrique, état structural...) (TARDIEU, 1984). Elles sont donc difficilement transposables d'un point à un autre.

Le but de cette étude est double : d'une part, acquérir des connaissances plus précises sur la façon dont se met en place le système racinaire du maïs au cours du cycle de végétation, d'autre part, examiner si certains paramètres plus étroitement liés au génotype de la plante conservent une certaine stabilité dans l'espace et le temps. Ainsi, le rythme d'apparition des racines primaires, l'apparition étant le moment où la racine devient visible sur la tige après éventuellement ablation des gaines foliaires susceptibles de la masquer, sera comparé à celui des feuilles.

Une analyse de la variabilité de ces paramètres en relation avec les variétés ou les conditions de milieu fera l'objet d'un $2^{\mathrm{e}}$ article, tandis que l'étude des phénomènes de croissance, par comparaison des évolutions des masses respectives du système aérien et du système racinaire, sera faite ultérieurement.

\section{DISPOSITIF EXPÉRIMENTAL}

L'expérimentation a été réalisée sur des parcelles de mais conduites selon les normes de la grande culture pendant 2 années successives, en 1982 et 1983, au Centre INRA de Colmar, de façon à disposer de suffisamment de plantes pour les observations et à mesurer non seulement des valeurs moyennes mais leurs dispersions in situ.

Le sol (tabl. 1) a une texture "de limon argileux" (La) ; il est normalement pourvu en matière organique, de $\mathrm{pH}$ modérément alcalin, formé sur loess. La teneur en bases échangeables est suffisante, celles en azote et phosphore légèrement déficientes.

Le champ expérimental, labouré à l'automne, est repris par hersage vers le 15 avril, après épandage d'un insecticide (lindane + chlorpyriphos-éthyl) et de la fertilisation phospho-potassique $(152 \mathrm{~kg}$ de $\mathrm{P}$ et $290 \mathrm{~kg}$ de $\mathrm{K}$ par ha en 1982 , pour corriger les faibles teneurs observées; $46 \mathrm{~kg}$ de $\mathrm{P}$ et $116 \mathrm{~kg}$ de $\mathrm{K}$ par ha en 1983). Le semis (21 avril $1982 ; 30$ avril 1983) est réalisé de façon à obtenir une densité réelle de 75000 pieds. ha $^{-1}$ (75 cm entre lignes). L'azote est apporté en 2 fois, après levée $\left(70 \mathrm{~kg}\right.$. ha ${ }^{-1}$ en 1982 ; $50 \mathrm{~kg} \cdot \mathrm{ha}^{-1}$ en 1983) et au stade 8 feuilles $\left(70 \mathrm{~kg} . \mathrm{ha}^{-1}\right.$ en $1982 ; 105 \mathrm{~kg} . \mathrm{ha}^{-1}$ en 1983 ).
TABLEAU 1

Caractéristiques du sol sur lequel ont été conduits les essais (couche labourée); la réserve en eau est d'environ $200 \mathrm{~mm}$ sur $1,50 \mathrm{~m}$. Soils characteristics of the experimental field.

\begin{tabular}{|c|c|c|c|}
\hline $\begin{array}{c}\text { Analyse physique } \\
\text { texture }(\%)\end{array}$ & & $\begin{array}{c}\text { Analyse chimique } \\
(\% / \%)\end{array}$ & \\
\hline Argile & 22,5 & Carbone organique & 11,1 \\
\hline Limon fin & 30,9 & Azote total (Kjeldahl) & 1,3 \\
\hline Limon grossier & 41,5 & Phosphore (Joret-Hébert) & 0,10 \\
\hline Sable fin & 0,4 & Potassium échangeable & 0,20 \\
\hline Sable grossier & 2,8 & Calcium échangeable & 7,55 \\
\hline \multirow[t]{2}{*}{ Calcaire total } & 14,7 & Magnésium échangeable & 0,16 \\
\hline & & $\mathrm{pH}$ & 8,4 \\
\hline
\end{tabular}

Le climat de Colmar est de type semi-continental, avec un maximum de pluies en été (SCHENCK, 1977). Des conditions très différentes ont été observées durant les 2 années d'essai que traduisent les écarts à la normale (moyenne 1895-1982) de la température et de la pluviométrie (fig. 1).

Les observations ont été conduites sur la variété (hybride simple) « Dea ». Cinq parcelles ont été délimitées dans le champ expérimental, comprenant chacune 2 lignes sur lesquelles ont été faites les prélèvements et 1 ligne de bordure de part et d'autre. Du semis à la récolte $(10$ octobre $1982 ; 19$ septembre 1983), 2 pieds par ligne (soit 20 pieds au total) ont été prélevés à la bêche avec une motte de terre d'environ $20 \times 20 \times 20 \mathrm{~cm}, 2$ fois par semaine jusqu'à la floraison, puis de façon plus espacée jusqu'à la récolte. La terre ayant été éliminée par aspersion, les observations et les mesures suivantes ont été effectuées :

- sur le système aérien : stade de développement, hauteur de la plante (prise du grain à l'extrémité de la feuille la plus longue), nombre de feuilles visibles (dès qu'elles émergent du cornet) et de feuilles étalées (ligule dégagée) ;

- sur le système racinaire : nombre de racines séminales; nombre d'entre-nœuds portant des racines adventives notés $\mathrm{E}$ suivis de leur numéro de rang, décomptés depuis le nœud scutellaire (KIESSELBACH, 1949) ; par entre-nœud, nombre de racines nodales primaires, classées en 2 catégories, celles de longueur inférieure à $3 \mathrm{~cm}$ et celles de longueur supérieure à $3 \mathrm{~cm}$; diamètre des racines primaires.

Pour ce qui concerne les racines primaires, les observations effectuées ont pour objet de distinguer celles nouvellement apparues (de la date où elles deviennent visibles sur la tige à celle où leur longueur dépasse $3 \mathrm{~cm}$ ) et celles plus âgées. Toutefois, l'étude montrera que les racines émises aux entre-nœuds supérieurs ( $E$ 8 et $E$ 9) restent à l'état d'ébauches (longueur $<3 \mathrm{~mm}$ ) de leur date d'apparition à la récolte. Elles ont donc été comptabilisées séparément des racines qui se sont allongées.

Selon une terminologie justifiée précédemment (PICARD, 1976), les racines nodales primaires sont celles issues de la base d'un entre-nœud de la tige à partir de $\mathrm{E} 2$. Les racines issues du mésocotyle $\mathrm{E} 1$ ont été comptabilisées parmi les séminales comme usuelle- 

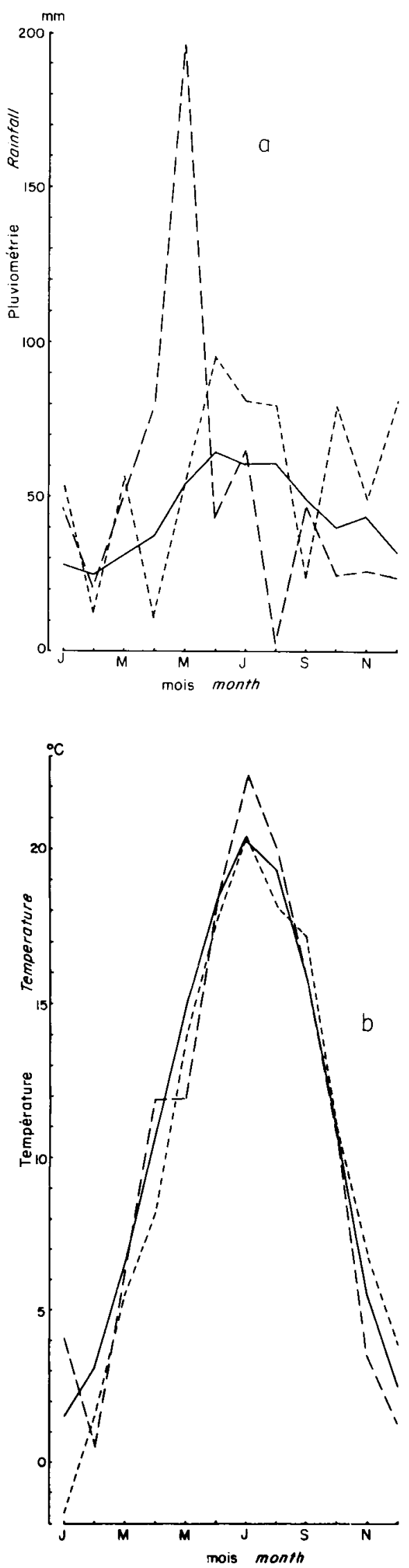

Figure 1

Pluviométrie moyenne annuelle (a) et température moyenne mensuelle (b) à Colmar.

Mean monthly rainfall (a) and mean monthly temperature (b) at Colmar.

Moyenne 1895-1982 Mean

1982

1983. ment, alors qu'en réalité il s'agit également de racines nodales primaires (ONDERDONK \& KETCHESON, 1972).

\section{RÉSULTATS}

Trois séries de données ont été ainsi recueillies ; celles ayant trait à la structure du système aérien seront examinées d'abord, pour comprendre les suivantes : celles caractéristiques de la structure de l'enracinement et enfin celles liées à la dynamique racinaire.

\section{A. Données de structure du système aérien}

La courbe d'évolution du nombre de feuilles (fig. 2) en fonction des sommes de températures de l'air exprimées en base $6^{\circ}$ (BLOC et al., 1983) montre une nette différence de développement des plantes entre les 2 années, qui n'est apparemment pas imputable à l'effet de la température. Ainsi, le nombre total de feuilles s'établit à 16,2 en 1982 et 15,7 en 1983 .

Le fait de prendre les températures du sol (sol nu ou sol enherbé) à - $10 \mathrm{~cm}$ ne modifie pas les différences observées.

Les dates d'apparition des inflorescences mâles (50 p. 100 de panicules visibles), le 8 juillet 1982 et le 11 juillet 1983, sont proches, exprimées en degrésjours ( $720 \mathrm{~d}$.j. en $1982,710 \mathrm{~d}$.j. en 1983), mais celles des inflorescences femelles apparaissent plus éloignées (790 d.j. en 1982, 870 d.j. en 1983). Toutefois, l'écart est peut-être exagéré par le fait que les observations n'ont été faites que tous les 3 ou $4 \mathrm{j}$, ce qui peut décaler la date exacte de floraison d'environ 40 à $60 \mathrm{~d} . \mathrm{j}$.

Les récoltes ont été effectuées le 10 octobre 1982 et le 19 septembre 1983, les rendements en grains (exprimés en matière sèche) s'élevant à $82 \mathrm{q} \cdot \mathrm{ha}^{-1}$ et 84 q. ha ${ }^{-1}$.

Il est intéressant de voir si les écarts constatés ont une répercussion sur la structure et la dynamique du système racinaire.

\section{B. Données de structure de l'enracinement}

La structure de l'enracinement résulte de la cinétique d'apparition des racines primaires puis de leur ramification. Il s'avère cependant plus simple d'exposer en premier les données de structure pour mieux comprendre la cinétique.

Les observations effectuées ont permis de constater que le diamètre des racines nodales primaires augmente linéairement avec le numéro de l'entre-nœud qui les porte (tabl. 2) et donc d'affecter sans erreur les racines à leurs entre-nœuds d'origine lorsque ceux-ci sont difficiles à distinguer ( $\mathrm{E} 2$ à $\mathrm{E} 4$ pour quelques plantes dans certains lots). Ils ne commencent en effet à s'allonger - c'est-à-dire que leur hauteur ne devient sensiblement supérieure au diamètre des racines qu'ils portent - qu'à partir du numéro 7 .

Des études de fréquence relative du nombre de racines par entre-nœud ont été faites en prenant en compte l'ensemble des plantes prélevées depuis le jour où l'émission est terminée pour cet entre-nœud (cf. $\S$ III C) - concrètement, dès que le nombre maximum de racines est atteint - jusqu'à la récolte. Ainsi, par exemple, pour le nombre de racines portées par E 2 (fig. 3), 20 lots de 20 plantes sont utilisables en 1982 ; sur les 400 plantes ainsi observées, 59 p. 100 


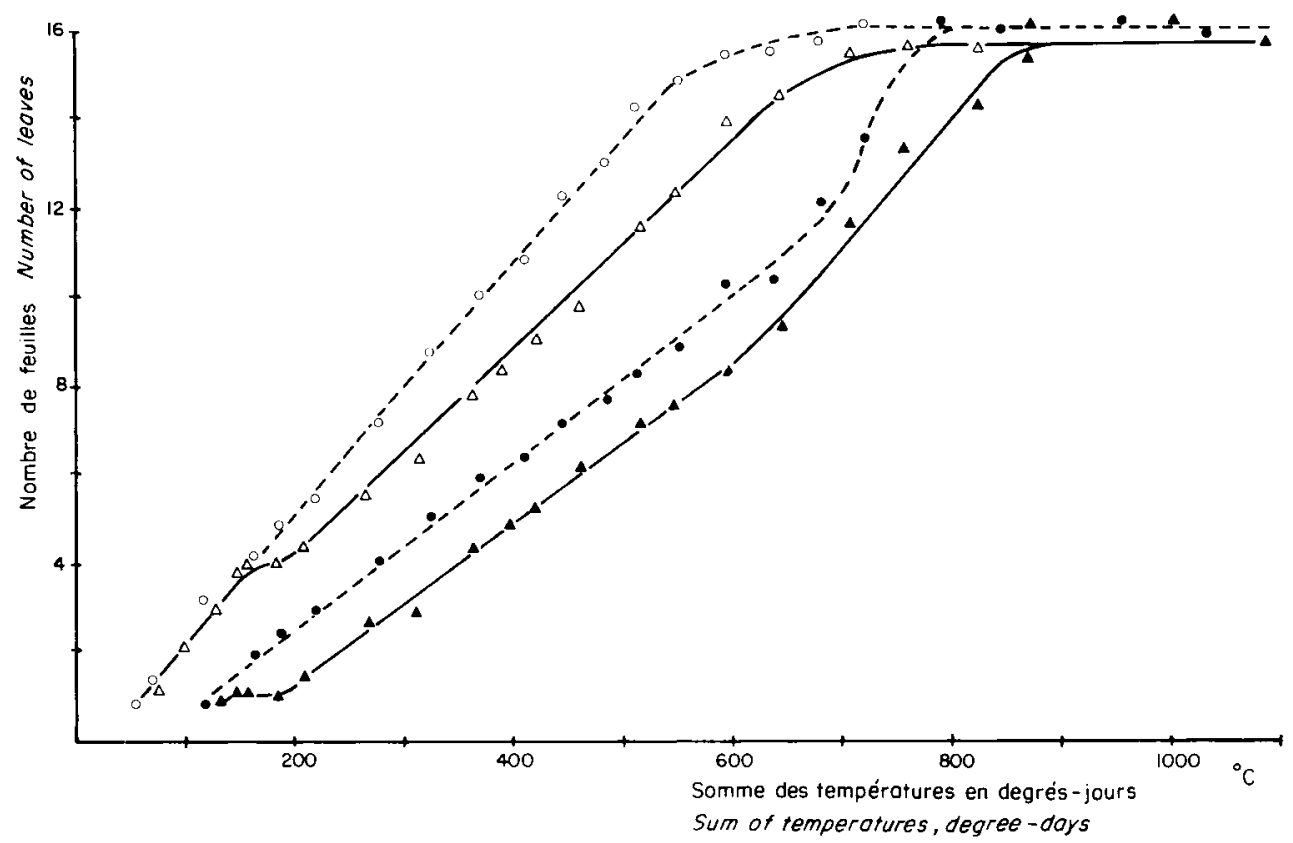

Figure 2

Nombre de feuilles (visibles ou "étalées », c'est-à-dire ligule visible) en fonction de la somme des températures.
Number of leaves (visible or «fully expanded", i.e. ligule visible) in relation to sum of temperatures (degree. days).

- 1982, 1983 : feuilles visibles, visible leaves

- 1982, \ 1983 : feuilles "étalées ", fully expanded leaves. ont 4 racines primaires et 87 p. 100,3 ou 4 racines primaires.

Le nombre de racines séminales primaires est statistiquement significativement plus élevé en 1982 qu'en 1983. Celui des racines nodales primaires par entrenœud diminue de E 2 à E 4, puis augmente, aussi bien en 1982 qu'en 1983. Il est statistiquement significativement plus élevé en 1982 pour E 3, E 5 et E 6 et plus faible pour $\mathrm{E} 8$; il y a donc un déficit de racines en 1983 par rapport à 1982 en début de croissance, compensé à la floraison, comme pour les feuilles.

A la fin du cycle du maïs, toutes les racines des entrenœuds 2 à 7 se sont allongées (d'au moins $3 \mathrm{~cm}$ ) et celles émises au-dessus du sol y ont pénétré ; une faible part des racines issues de l'entre-nœud 8 restent

\section{TABLEAU 2}

Variations du diamètre moyen des racines nodales primaires en fonction du numéro de l'entre-noud qui les porte.

Variations of the mean diameter of the nodal primary roots in relation to the rank of the internode by which there are born. $D=0,93 N E-1,08 \quad r=0,99 * * \quad(5 \mathrm{dl})$

$D^{\prime}=1,25 N E-1,80 \quad r=0,98 * *(4 \mathrm{dl})$

\begin{tabular}{|c|c|c|c|c|}
\hline \multirow[t]{2}{*}{$\begin{array}{l}\text { Numéro de } \\
\text { l'entrenœud } \\
\text { NE }\end{array}$} & \multicolumn{2}{|c|}{$\begin{array}{l}\text { Diamètre } D \\
\text { (pris à environ } 6 \mathrm{~cm} \text { de } \\
\text { l'extrémité basale 1982) } \\
\mathrm{mm}\end{array}$} & \multicolumn{2}{|c|}{$\begin{array}{c}\text { Diamètre } \Gamma^{\prime} \\
\text { (pris à l'extrémit } \\
\text { basale } 1983 \text { ) } \\
\text { mm }\end{array}$} \\
\hline & moyenne & écart-type & moyenne & écart-type \\
\hline 2 & 1,2 & 0,15 & 1,5 & 0,14 \\
\hline 3 & 1,6 & 0,18 & 1,6 & 0,19 \\
\hline 4 & 2,3 & 0,26 & 2,8 & 0,46 \\
\hline 5 & 3,4 & 0,71 & 4,0 & 0,76 \\
\hline 6 & 4,6 & 0,75 & 5,6 & 0,90 \\
\hline 7 & 5,6 & 0,85 & 7,4 & 1,23 \\
\hline 8 & 6,5 & 1,06 & & \\
\hline
\end{tabular}

aériennes. Par contre, celles provenant des entrenœuds 9 et 10 restent à l'état d'ébauches, de quelques $\mathrm{mm}$ de long au plus (fig. 3). Elles sont au nombre de 16 en moyenne à l'entre-nœud 9. Elles ne sont pas toujours présentes à l'entre-nœud 10:10 p. 100 seulement des plantes en ont en 1982, 60 p. 100 en 1983 et leur nombre est alors très variable.

La dispersion des valeurs varie avec les entre-nœuds et les années. Elle est relativement faible pour $\mathrm{E} 2$, puis croît. De E 3 à E 5, les coefficients de variation, relativement comparables en 1982 et 1983 , oscillent entre 27 et 39 p. 100. De E 6 à E 8, les résultats des 2 années divergent : en 1982, le coefficient de variation continue à osciller entre les limites précédentes, alors que, en 1983, il baisse de façon marquée, l'écarttype augmentant peu relativement à la moyenne. Ceci paraît s'expliquer par le fait qu'en 1982, certaines plantes n'avaient pas de racines aux entre-nœuds E 7 et $E$ 8, ce qui n'est pas le cas en 1983. Pour E 9 et E 10, l'analyse offre peu d'intérêt, les racines ne s'allongeant pas.

\section{Cinétique d'apparition des racines nodales primaires}

Les courbes d'évolution du nombre de racines nodales primaires par entre-nœud en fonction du temps (fig. 4) montrent que l'émission se produit successivement entre-nœud par entre-nœud et que, pour chacun d'entre eux, il est possible de définir une durée d'émission. Celle-ci, prise arbitrairement comme égale à l'intervalle séparant la date d'apparition de la $1^{\text {re }}$ racine visible de celle où le nombre maximum de racines est atteint, est relativement brève.

Le synchronisme de la sortie des racines d'un entrenœud et le décalage entre celles de 2 entre-nœuds successifs peut s'apprécier de façon plus détaillée à partir 
1982

1983

1982
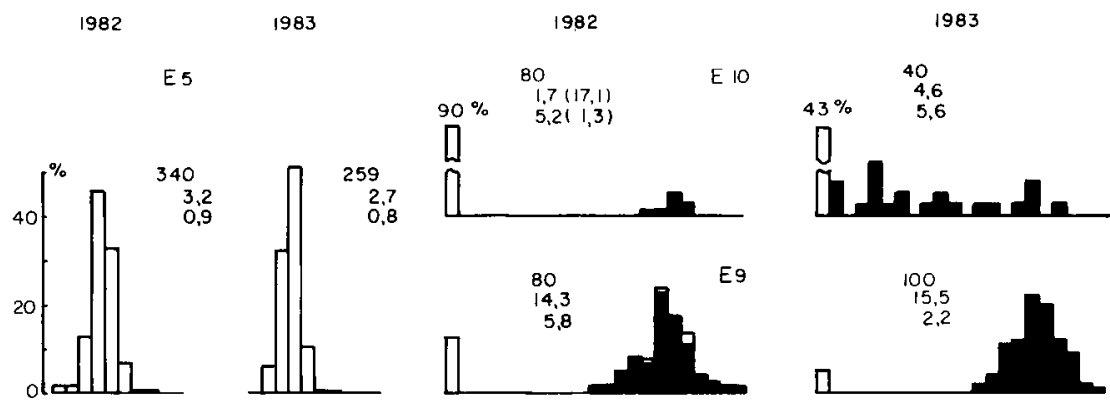

$\square$
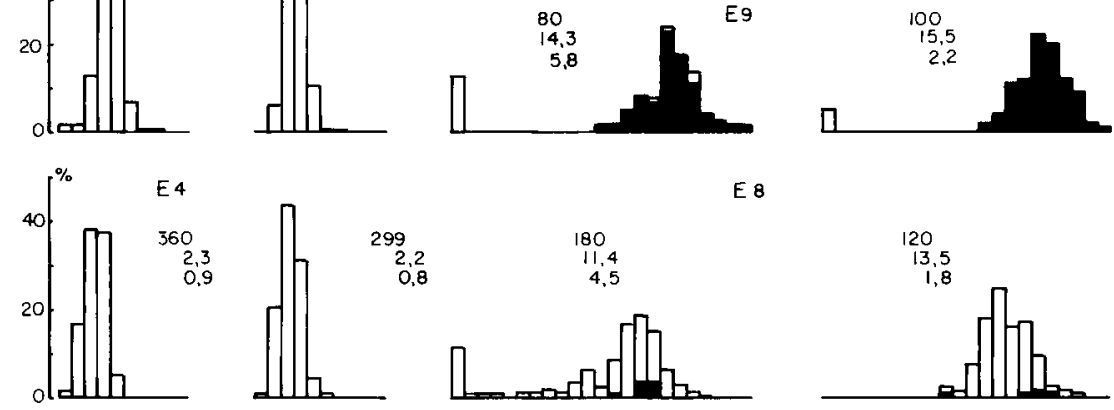

E 8
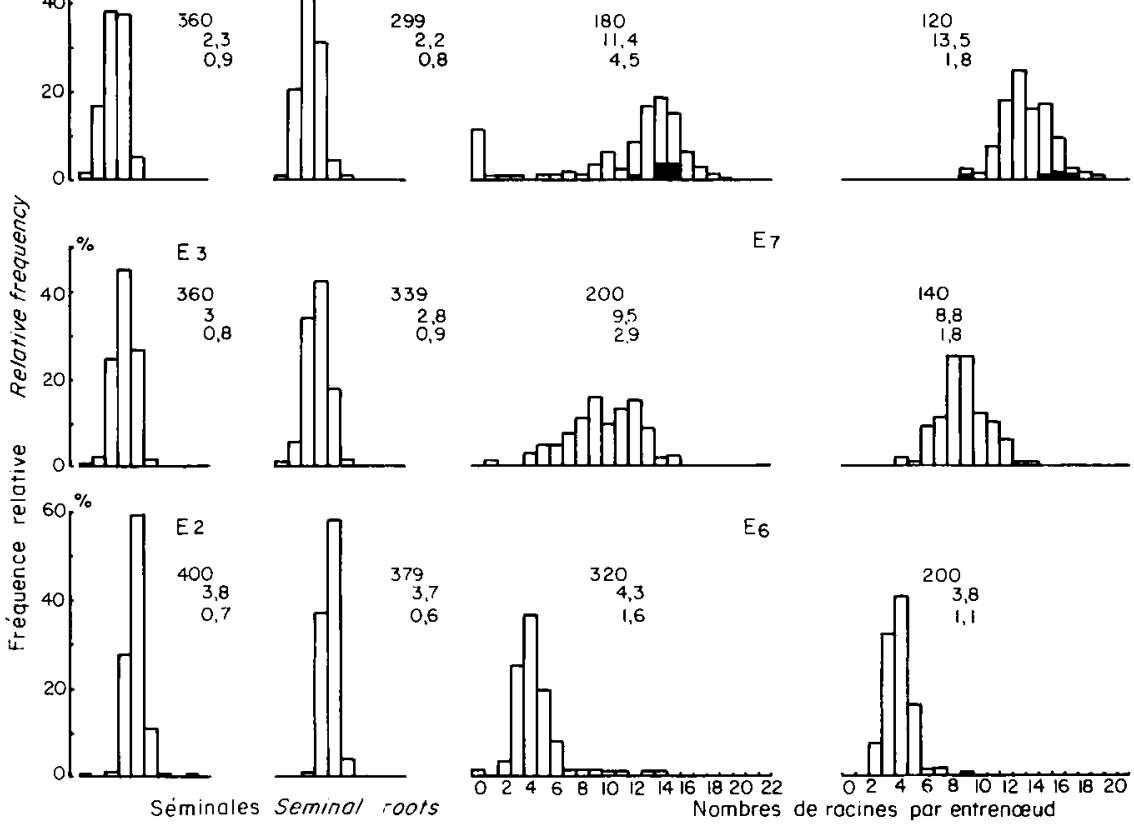

$E_{7}$
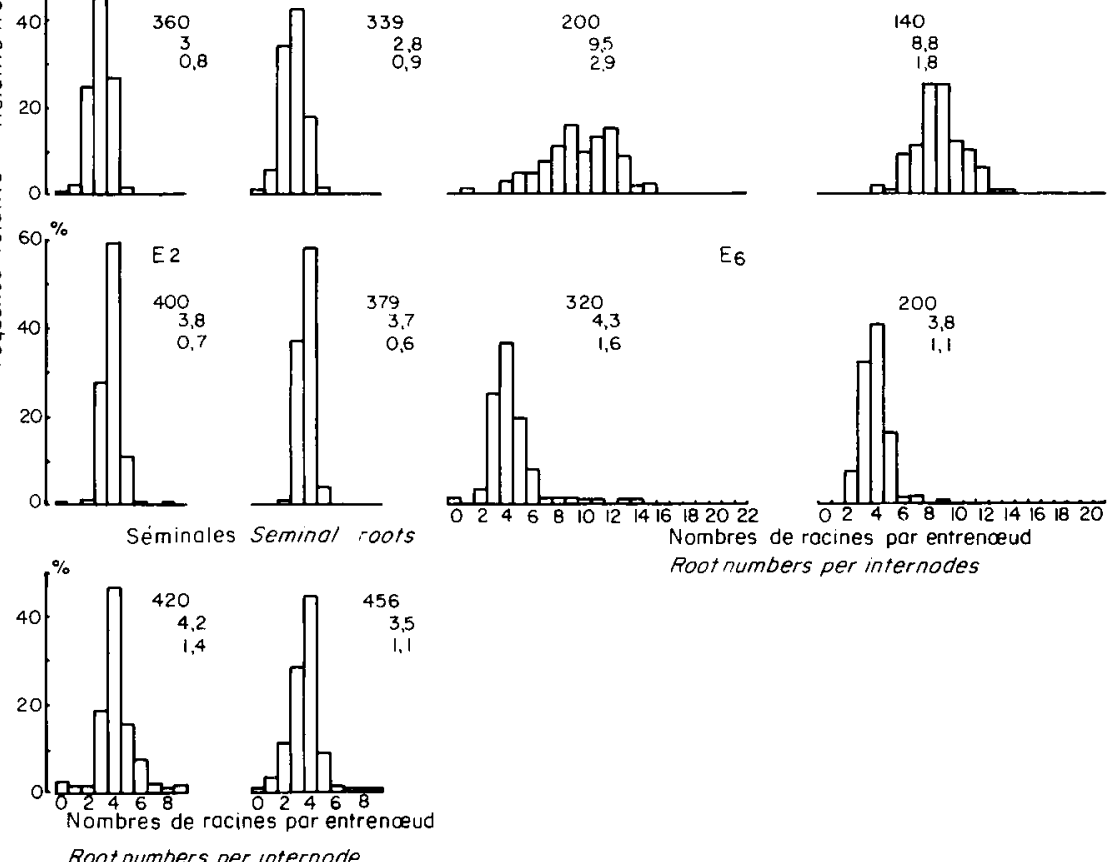

Figure 3

: ébauches racinaires; short roots

Distribution des nombres de racines primaires par entrenoud, selon les années

$E 2$ : entrenoud $n^{\circ} 2$; internode $n^{\circ} 2$

400 : effectif de la population analysée; number of plants under study

Distribution of the numbers of primary roots per internode, according to years.

3,8 : moyenne; mean

0,7 : écart-type; standard error.

des résultats sur les racines de longueur inférieure à $3 \mathrm{~cm}$ visibles à un entre-nœud donné. Au tableau 3 figurent pour chaque entre-nœud, la date d'apparition de la $1^{\text {re }}$ racine visible, puis celle à laquelle le nombre maximum moyen est atteint, enfin celle à laquelle toutes les racines ont une longueur supérieure à $3 \mathrm{~cm}$. Le décalage entre ces 2 dernières dates - qui n'est pas systématique - résulte de l'écart entre 2 mesures $(3$ ou 4 jours) et de la variabilité entre plantes. Pour les entrenœuds 2 à 6 , la durée de l'émission, calculée à partir de $J_{1}$, pratiquement la même pour les 2 années, est de 7 à $8 \mathrm{j}$ (un peu plus longue pour les entre-nœuds 2, 3 et 6 , un peu plus courte pour les entre-nœuds 4 et 5 ). Cette durée augmente pour les entre-nœuds 7 et 8 et est 2 fois plus longue en 1982 qu'en 1983, ce qui fait qu'à $75 \mathrm{j}$ les plantes ont le même nombre de racines primaires, 40 environ, alors qu'entre le $25^{\mathrm{e}}$ et le $75^{\mathrm{e}} \mathrm{j}$, les plantes de 1983 avaient un certain retard sur celles de 1982.

Pour tenter d'expliquer les différences observées entre les résultats des 2 années, on peut substituer à l'échelle de temps astronomique soit des degrés-jours, soit le phyllochrone (Gounot et al., 1980).

L'utilisation des degrés-jours pour étudier la variation du nombre cumulé de racines nodales primaires (fig. 5) ne permet pas de regrouper les résultats des 2 années sur une même cinétique (comme pour le système aérien) : en 1983, la sortie des racines issues de l'entre-nœud 3 a été considérablement retardée par les événements climatiques de la fin du mois de mai. 


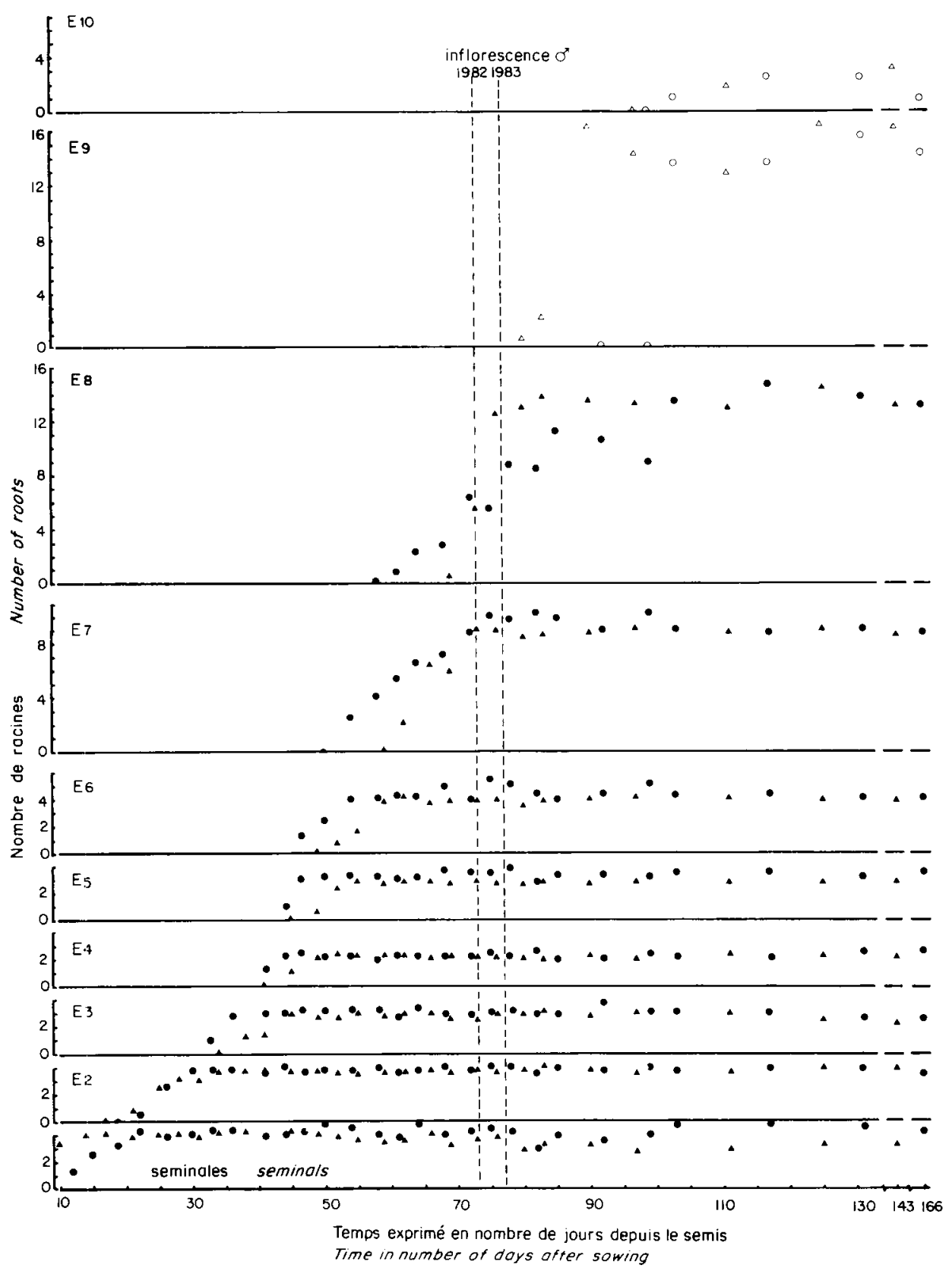

Figure 4

Evolution du nombre de racines séminales ou nodales primaires, entrenoud par entrenceud, en fonction du temps.

Evolution of the number of seminal or nodal primary roots per internode, in relation to time.

L'écart, qui apparaît dès le début du cycle, se maintient jusqu'à la floraison.

L'évolution annuelle du nombre cumulé de racines nodales primaires en fonction des degrés-jours présente à certaines époques des changements de pente et des paliers du fait que l'émission des racines ne se produit pas de façon régulière mais, au contraire, par vagues successives: l'augmentation du nombre de racines par entre-nœud à partir du $5^{\mathrm{e}}$ entre-nœud se traduit par des accroissements de pente; les paliers correspondent aux intervalles relativement longs qui parfois séparent l'arrêt de l'émission à un entre-nœud du début de celle à l'entre-nœud suivant (cas de l'émission en E 2 en 1982 et surtout en 1983, puis après celle en $\mathrm{E} 6$, pour les 2 années).
- 1982, $\Delta 1983$ : ébauches, short roots

- 1982, \& 1983 : racines; roots.

E 2 : entrenoud $n^{\circ} 2$; internode $n^{\circ} 2$.

Par contre, si l'on rapporte le nombre de racines nodales émises par entre-nœud au phyllochrone, ce qui impose de s'arrêter à la floraison (E 8), les résultats des 2 années (fig. 6) se rapprochent sensiblement. Les déviations qui subsistent peuvent s'expliquer en partie par le fait que les effectifs de plantes ayant un stade et un nombre de racines données deviennent très variables. Il reste cependant un écart statistiquement significatif entre les 2 années pour E 3.

L'évolution du nombre total de racines nodales primaires est étroitement liée à celle du nombre total de feuilles. Sur l'ensemble des données, on met en évidence une relation d'allométrie (fig. 7), classiquement observée lors de la comparaison des croissances d'organes d'une même plante. 
TABLEAU 3

Dates de début et de fin ainsi que durée effective, exprimées en jours, de l'émission des racines primaires entre-nœud par entre-nœud. Dates of beginning and of end and real duration, in days, of the emission of the primary roots, per internode.

$D$ : début de l'émission (l'es racines visibles) en jours depuis le semis beginning of the emission (first roots appearing), in days from sowing

$M$ : date à laquelle le nombre maximum moyen de racines est atteint pour l'entre-noud considéré, en jours depuis le semis date at which the maximum mean number of roots is reached for the internode under consideration, in days from sowing

$F$ : date d'apparition des dernières racines de longueur inférieure à $3 \mathrm{~cm}$, en jours depuis le semis appearance date of the last roots (length $<3 \mathrm{~cm}$ ), in days from sowing

$J_{l}$ : durée de l'intervalle $M-D ; J_{2}$ : durée de l'intervalle $F-D$, en jours $M-D$ interval $J_{2}: F-D$ interval, in days.

\begin{tabular}{|c|c|c|c|c|c|c|c|c|}
\hline & & \multicolumn{7}{|c|}{ Système nodal. Entre-nœuds : } \\
\hline & & E 2 & E 3 & E 4 & E 5 & E 6 & E 7 & E 8 \\
\hline \multirow[t]{4}{*}{1982} & $\mathrm{D}$ & 22 & 33 & 36 & 44 & 47 & 54 & 58 \\
\hline & $\mathbf{M}$ & 30 & 41 & 44 & 47 & 58 & 75 & 85 \\
\hline & $\mathrm{F}$ & 41 & 44 & 44 & 47 & 68 & 85 & récolte \\
\hline & $\mathrm{J}_{1} ; \mathrm{J}_{2}$ & $8 ; 19$ & $8 ; 11$ & 8 & 3 & $11 ; 21$ & $21 ; 31$ & 27 \\
\hline \multirow[t]{4}{*}{1983} & $\mathrm{D}$ & 21 & 38 & 45 & 49 & 52 & 62 & 69 \\
\hline & $\mathrm{M}$ & 34 & 45 & 49 & 55 & 62 & 73 & 83 \\
\hline & $\mathrm{F}$ & 38 & 45 & 49 & 55 & 66 & 76 & récolte \\
\hline & $\mathrm{J}_{1} ; \mathrm{J}_{2}$ & $13 ; 17$ & 7 & 4 & 6 & $10 ; 14$ & $11 ; 14$ & 14 \\
\hline
\end{tabular}

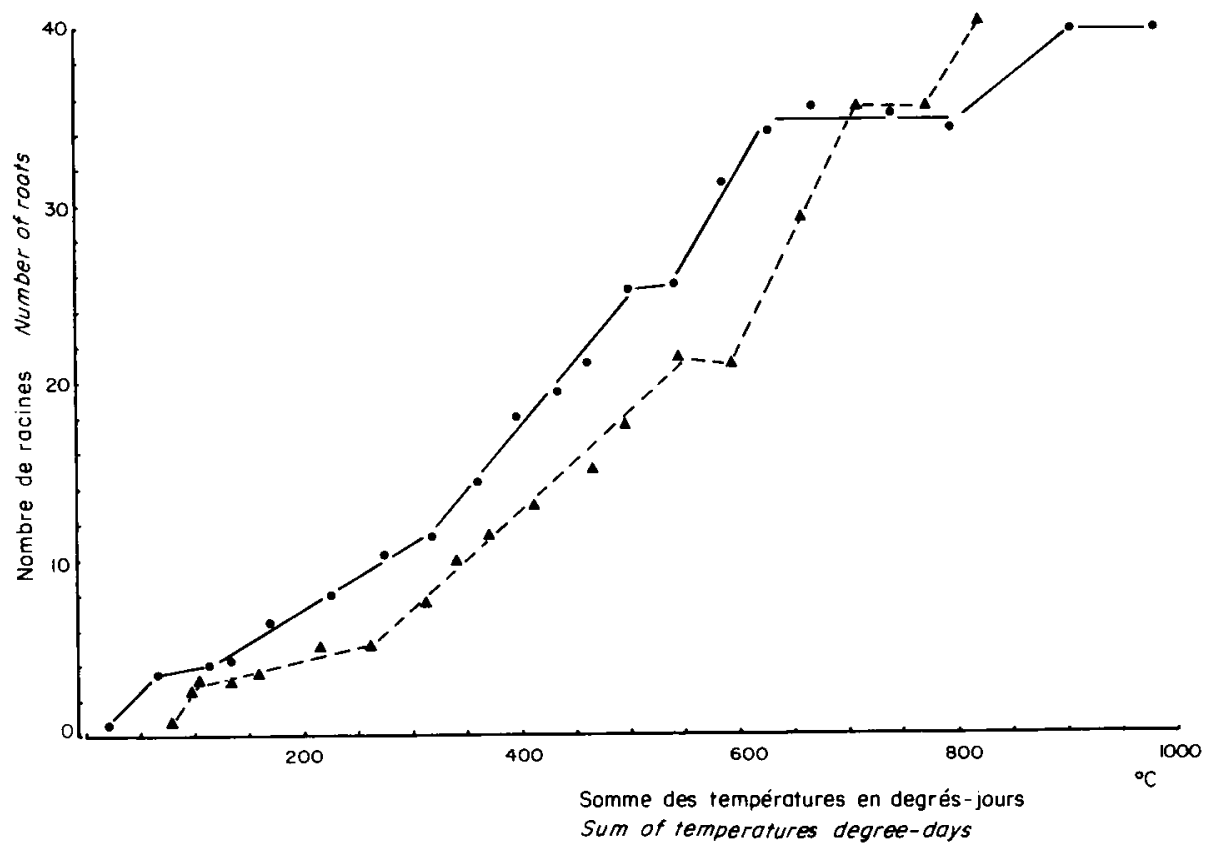

Figure 5

Nombre cumulé de racines primaires nodales en fonction de la somme des températures.
Cumulative number of nodal primary roots in relation to the sum of temperatures.

- $1982 ; \Delta 1983$.
Toutefois, malgré la qualité de l'ajustement statistique, un certain écart subsiste entre le modèle théorique et les données à partir de l'apparition de la feuille 6. Il est donc préférable de les ajuster à 3 modèles linéaires successifs (fig. 7), en raison de l'augmentation du nombre de racines nodales par entre-nœud à partir de E 5. Durant les premiers phyllochrones, il est même possible de distinguer 2 sous-modèles légèrement décalés pour 1982 et 1983 , ce qui confirme les différences déjà observées entre les 2 années.
La coordination des émissions foliaire et racinaire est également révélée par l'étude des dates de début et de fin d'émission des racines nodales primaires par entre-nœud, exprimées en temps phyllochronique (fig. 8). Les expressions de ces dates sont des fonctions linéaires des nombres d'entre-nœuds ; la relation réciproque permet de montrer que la durée de l'émission pour un entre-nœud donné est, en moyenne, constante et égale à 2 phyllochrones. 

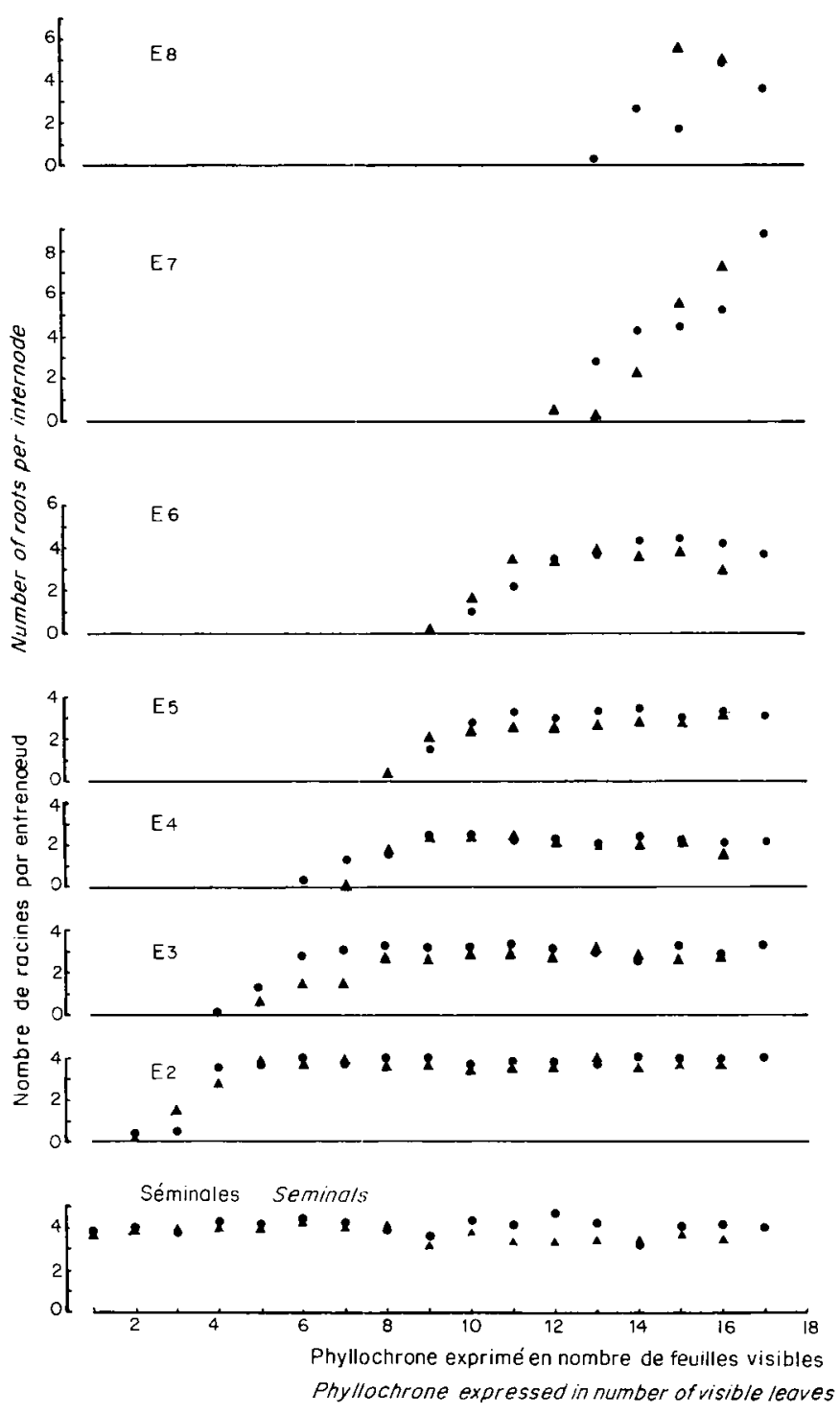

Figure 6

Evolution du nombre de racines séminales et nodales primaires, entrenceud par entrenceud, en fonction du phyllochrone.

Evolution of the number of seminal and nodal primary roots per internode, in relation to the rank of the internode and the phyllochrone.

E 2 : entrenoud $n^{\circ} 2$; internode $n^{\circ} 2$

- $1982 ; \boldsymbol{\Delta} 1983$.

Les équations correspondantes s'expriment par :

début de l'émission à l'entre-nœud $\mathrm{E}$ :

$\mathrm{E}=0,51 \mathrm{NF}+0,71 \quad \mathrm{r}=0,99 * * 14 \mathrm{dl}$;

fin de l'émission au même entre-nœud $\mathrm{E}$ :

$\mathrm{E}=0,48 \mathrm{NF}-0,18 \quad \mathrm{r}=0,99^{* *} \quad 14 \mathrm{dl}$.

Toutefois, un examen entre-nœud par entre-nœud et année par année révèle une certaine variabilité de la durée de l'émission avec des écarts importants pour E 2 et E 4. S'agit-il d'une variabilité aléatoire ou au contraire révélatrice de l'effet de certains facteurs qui restent à identifier?

\section{DISCUSSION ET CONCLUSION}

Le maïs constitue un matériel intéressant pour étudier le rythme d'apparition des racines: en effet,

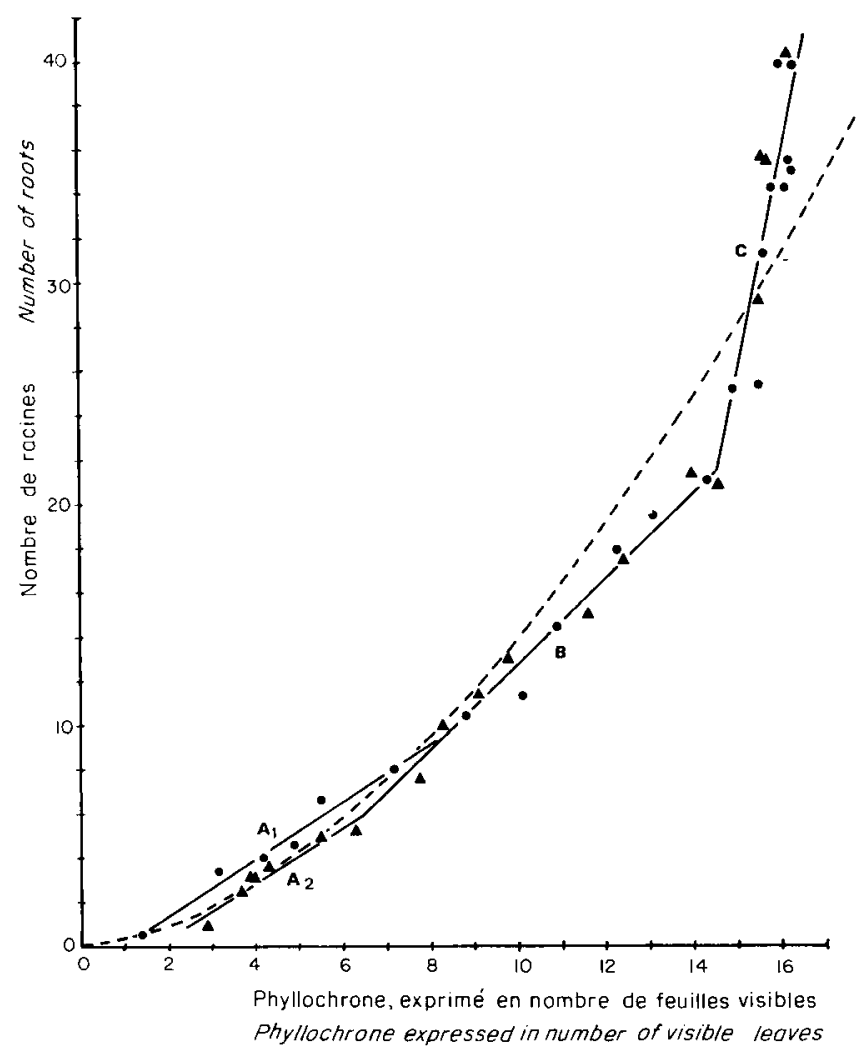

Figure 7

Evolution du nombre de racines primaires nodales en fonction du phyllochrone.

Evolution of the number of nodal primary roots in relation to the phyllochrone.

- 1982 ; $\Delta 1983$.

----- Modèle bilogarithmique Bilogarithmic model :

$$
\begin{aligned}
& \operatorname{LnNR}=1,73 \operatorname{LnNF}-1,35 \quad r=0,98^{* *} \\
& \text { Modèles linéaires Linear regression : } \\
& A_{1}(1982) \quad N R=1,29 N F-1,22 \quad r=0,98^{* *} \\
& A_{2} \text { (1983) } \quad N R=1,25 N F-2,18 \quad r=0,98^{* *} \\
& A_{1}+A_{2} \quad N R=1,23 N F-1,61 \quad r=0,95 * * \\
& { }_{B} \quad N R=1,94 N F-6,70 \quad r=0,98 * * \\
& \begin{array}{lll}
B & N R=9,74 N F-1,20 & r=0,98^{* *}
\end{array} \\
& \text { NR: Nombre cumulé de racines nodales primaires. } \\
& \text { Cumulative number of nodal primary roots. } \\
& N F: \text { Nombre de feuilles } \\
& \text { Leaf number. }
\end{aligned}
$$

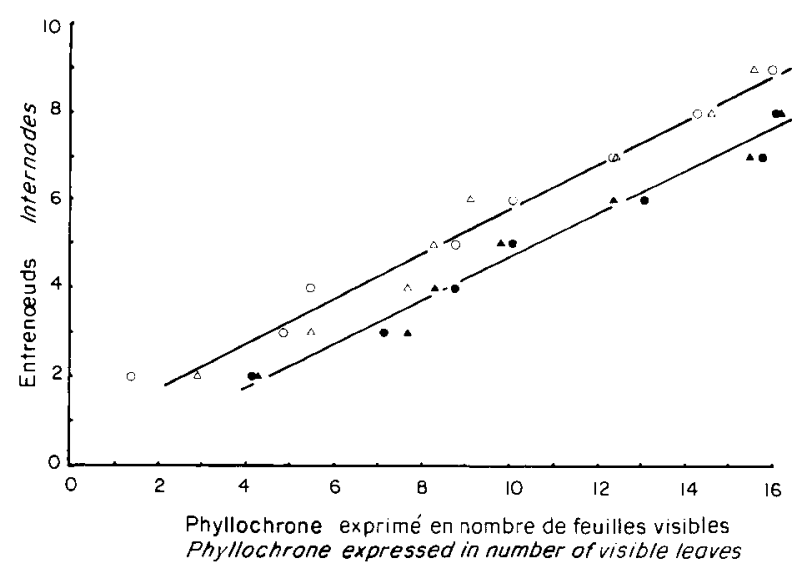

Figure 8

Dates de début et de fin de l'émission des racines nodales primaires, exprimées en temps phyllochronique, entrenceud par entrenœud.

Dates of the beginning and of the end of nodal primary root emission, expressed in phyllochronic time, per internode.

- 1982, $\Delta 1983$ : début d'émission ; beginning of emission.

- 1982, $\Delta 1983$ : fin d'émission ; end of emission. 
l'absence ou le caractère très limité du tallage, le diamètre croissant des racines nodales primaires d'un entre-nœud au suivant, facilitent le dénombrement de ces dernières et le repérage de leur origine.

Comme pour le riz (PICARD \& JACQUOT, 1976) ou le blé (PINTHUS, 1969 ; KLEPPER et al., 1984), les résultats font ressortir l'étroite liaison entre le développement du système aérien et celui du système racinaire. Cependant, la relation entre le nombre de feuilles et le nombre cumulé de racines nodales primaires n'est pas linéaire comme dans le cas du blé (KLEPPER et al., 1984), en raison du nombre variable de racines par entre-nœud. Curieusement, ce dernier commence par diminuer de l'entre-nœud 2 aux entre-nœuds 3 et 4, avant d'augmenter, encore que YAMAZAKI \& KAERIYAMA (1982) le trouvent constant.

Cette diminution pose un problème. En effet, et bien que l'initiation et l'émission des racines primaires semblent ne pas avoir été étudiées du point de vue morphogénétique au contraire de celles des racines secondaires (BELL \& McCULLY, 1970; KARAS \& McCULLY, 1973), il est vraisemblable que celles-ci proviennent de la différenciation de cellules situées à proximité des faisceaux vasculaires (MARTIN \& HARRIS, 1976). Le nombre de ces faisceaux devant aller en augmentant au fur et à mesure que le rang de l'entrenœud croît et donc que la tige s'épaissit (SHARMANN, 1944), il serait logique que le nombre de racines nodales primaires reste constant ou aille en augmentant avec le rang de l'entre-nœud.

D'autre part, si le nombre de racines émises au niveau de l'entre-nœud 2 paraît déterminé de façon assez stricte par le génotype, l'augmentation de la variabilité à partir de l'entre-nœud 3 et les écarts constatés entre années traduisent un rôle non négligeable de l'environnement sur le nombre de racines primaires et la cinétique d'émission.

Pour le nombre de racines, tout se passe comme s'il existait un potentiel que les facteurs et conditions de milieu permettaient ou non d'exprimer. S'il était constant et égal à 4 pour les entre-nœuds E 2 à E 5, cela signifierait que, même en 1982 et a fortiori en 1983, il n'a pas pu être extériorisé. A partir de E 6, il augmenterait fortement - il n'est pas possible de lui attribuer actuellement une valeur - permettant des phénomènes de compensation, par rapport à un déficit antérieur notamment.

En ce qui concerne la cinétique d'émission, les résultats montrent que les facteurs et conditions de milieu agissent davantage sur l'ensemble du développement de la plante - fait bien connu pour le développement du système aérien (BONHOMME, 1983) que sur les interactions développement du système aérien $\mathrm{x}$ développement du système racinaire. Ceci confirme les idées de Gounot et al. (1980) sur l'inté- rêt d'utiliser comme norme de temps le phyllochrone pour suivre la morphogenèse de la plante. Cette forte interaction apparaît très importante notamment en vue de la modélisation de sa croissance et de son développement, puisque cela signifie que l'on peut prévoir le rythme d'émission des racines nodales primaires à partir de celui des feuilles, sans toutefois présumer de la croissance ultérieure des racines émises : en effet, une relation étroite entre date d'émission, ou entre nombre d'organes, n'a pas nécessairement d'implication au niveau de la taille de ces organes.

A propos des relations nombre d'organes $\mathrm{x}$ taille de ces organes, il faut cependant noter que l'arrêt de l'émission des racines, qui se produit peu après la floraison, coïncide avec le moment où, selon la bibliographie, le maximum de matière sèche de l'enracinement est atteint (MENGEL \& BARBER, 1974). Cet arrêt a déjà été observé chez les céréales (PINTHUS, 1969 ; PICARD \& JACQUOT, 1976 ; KLEPPER et al., 1984) ou chez d'autres graminées (PICARD, 1976). Il pose le problème de l'évolution de l'activité racinaire dans la phase floraison-maturation du cycle, en relation notamment avec les variations d'humidité du sol durant cette phase.

Par ailleurs, les caractères morphologiques observés ne permettent pas de fonder la distinction classiquement faite entre racines nodales « normales » et racines nodales «d'ancrage " correspondant aux entrenœuds supérieurs (BONHOMME, 1983). Ceci confirme les résultats de MAERTENS (1970) qui ne met en évidence aucune variation d'absorption des éléments minéraux par les racines de maïs en relation ni avec leur diamètre ni avec l'entre-nœud dont elles proviennent. Cependant, LESAINT et al. (1979) notent des différences de composition chimique entre racines de faible diamètre et racines de fort diamètre, qu'ils expliquent par une activité métabolique accrue des grosses racines, notamment vis-à-vis des nitrates. Le problème mérite donc d'être approfondi.

Une autre question à élucider plus en détail est celle des interactions de croissance entre racines nodales primaires émises soit à un même entre-nœud soit à des entre-nœuds différents. BROUWER (1981) a montré qu'il y a des corrélations de croissance entre soussystème séminal et sous-système nodal pour de jeunes plantes de maïs. Mais le fait qu'on puisse définir une durée d'émission pour chaque entre-nœud implique l'existence de modes de régulation à analyser. Il faudrait également savoir si les racines émises ont des vitesses d'élongation - indépendamment des effets des facteurs liés au milieu - constantes ou liées au rythme d'apparition. 


\section{RÉFÉRENCES BIBLIOGRAPHIQUES}

Allmaras R. R., Nelson W. W., 1971. Corn (Zea mays L.) root configuration as influenced by some row-interrow variants of tillage and straw mulch management. Proc. Soil Sci. Soc. Am., 35, 974 980.

Bell J. K., McCully M. E., 1970. A histological study of lateral root initiation and development in Zea mays. Protoplasma, 70, 179-205.

Bloc D., Gay J. P., Gouet J. P., 1983. Durée des phases végétatives et reproductives chez le maïs : influence de la température. Comm Coll. Physiol. mais, Royan, 15-17/03/1983 (en cours d'impression).

Bonhomme R., 1983. Mise en place des appareils foliaire et racinaire chez le maîs. Comm. Coll. Physiol. mais, Royan, 15-17/03/ 1983 (en cours d'impression).

Brouwer R., 1981. Coordination of growth phenomena within a root system of intact maize plants. Plant Soil, 63, 65-72.

Garcia de Cortazar V., 1982. Modélisation de la croissance d'une culture de maiis. Thèse Doct. Ing., Univ. Paris-Sud, $111 \mathrm{p}$.

Girardin Ph., 1982. Essais de modélisations de la croissance et du développement du mais-grain. Thèse Doct. Ing., Univ. Paris-Sud, $145 \mathrm{p}$.

Gounot M., Atry M., N'Kandza J., Yu O., 1980. Photosynthèse nette et rythme d'apparition des feuilles chez le dactyle (Dactylis glomerata L.) C.R. Acad. Sci., Paris, 290 D, 1257-1260.

Karas I., McCully M. E., 1973. Further studies of the histology of lateral root development in Zea mays. Protoplasma, 77, 243-269.

Kiesselbach T. A., 1949. The structure and reproduction of corn. Univ. of Nebraska Press, rééd., 1980, 96 p.

Klepper B., Belford R. K., Rickman R. W., 1984. Root and shoot development in winter wheat. Agron. J., 76, 117-122.

Kundu S. S., Skogerboe G. V., Walker W. R., 1982. Improvement and sensitivity analysis of the CORNGRO model. Ecol. Modelling, 16, 209-239.

Lesaint C., Grandjean M., Coic Y., 1979. Comparaison de la composition minérale des petites et grosses racines d'une même plante (maïs et tomate). Déductions physiologiques et agronomiques. C. R. Acad. Agric. Fr., 65, 970-981.

Maertens C., 1970. Influence des conditions de milieu sur l'absorption de l'eau et des éléments minéraux par les systèmes radiculaires de quelques graminées cultivées. Thèse Doct. Ing., Univ. Toulouse, $162 \mathrm{p}$.

Martin E. M., Harris W. M., 1976. Adventitious root development from the coleoptilar node in Zea mays L. Am. J. Bot., 63, 890-897.

Mengel D. B., Barber S. A., 1974. Development and distribution of the corn root system under field conditions. Agron. J., 66, 341-344.

Onderdonk J. J., Ketcheson J. W., 1972. A standardization of terminology for the morphological description of corn seedlings. Can. J. Plant Sci., 52, 1003-1006.

Picard D., 1976. Dynamique racinaire de Panicum maximum Jacq. et apport au sol de matière organique. Thèse Doct. ès Sci., Clermont-Ferrand, $275 \mathrm{p}$.

Picard D., Jacquot M., 1976. Rythmes d'émission comparés des racines nodales de trois variétés de riz. Agron. Trop., 31, 151-169.

Pinthus M. J., 1969. Tillering and coronal root formation in some common and durum wheat varieties. Crop Sci., 9, 267-272.

Schenck Ch., 1977. Les conditions climatiques à Colmar de 1976 à 1978. Bull. Soc. Hist. Nat. Colmar, 56, 147-171.

Sebillotte M., 1974. Agronomie et agriculture : essai d'analyse des tâches de l'agronome. Cah. ORSTOM, sér. Biol., 24, 3-25.

Sharmann B. C., 1942. Developmental anatomy of the shoot of Zea mays L. Ann. Bot., 6, 245-282.

Tardieu F., 1984. Etude au champ de l'enracinement du mais. Influence de l'état structural sur la répartition des racines. Conséquences sur l'alimentation hydrique. Thèse Doc. Ing. INAPG, $232 \mathrm{p}$.

Yamazaki K., Kaeriyama N., 1982. The morphological characters and the growing directions of primary roots of corn plants. Jpn. J. Crop Sci., 51, 585-590. 